\title{
MAIN-SEQUENCE STARS MASQUERADING AS YOUNG STELLAR OBJECTS IN THE CENTRAL MOLECULAR ZONE
}

\author{
Christine M. Koepferl, Thomas P. Robitaille, Esteban F. E. Morales, and Katharine G. Johnston \\ Max Planck Institute for Astronomy, Königstuhl 17, D-69117 Heidelberg, Germany; koepferl@ mpia.de \\ Received 2014 July 13; accepted 2014 November 13; published 2015 January 15
}

\begin{abstract}
In contrast to most other galaxies, star formation rates in the Milky Way can be estimated directly from young stellar objects (YSOs). In the central molecular zone the star formation rate calculated from the number of YSOs with $24 \mu \mathrm{m}$ emission is up to an order of magnitude higher than the value estimated from methods based on diffuse emission (such as free-free emission). Whether this effect is real or whether it indicates problems with either or both star formation rate measures is not currently known. In this paper, we investigate whether estimates based on YSOs could be heavily contaminated by more evolved objects such as main-sequence stars. We present radiative transfer models of YSOs and of main-sequence stars in a constant ambient medium which show that the main-sequence objects can indeed mimic YSOs at $24 \mu \mathrm{m}$. However, we show that in some cases the main-sequence models can be marginally resolved at $24 \mu \mathrm{m}$, whereas the YSO models are always unresolved. Based on the fraction of resolved MIPS $24 \mu \mathrm{m}$ sources in the sample of YSOs previously used to compute the star formation rate, we estimate the fraction of misclassified "YSOs" to be at least 63\%, which suggests that the star formation rate previously determined from YSOs is likely to be at least a factor of three too high.
\end{abstract}

Key words: Galaxy: center - radiative transfer - stars: formation - stars: pre-main sequence - stars: protostars

Supporting material: machine-readable table

\section{INTRODUCTION}

Various indirect techniques based on diffuse emission are traditionally applied in order to measure the star formation rate (SFR) of galaxies throughout the universe. These common techniques use star formation tracers such as free-free centimeter continuum, $\mathrm{H} \alpha$, and far-infrared (FIR) emission to indirectly infer the rate of forming stars (for details, see review by Calzetti 2013). Free-free emission (or bremsstrahlung) emitted from ionized electrons, and recombination lines of ionized hydrogen (e.g., Balmer series $\mathrm{H} \alpha$ ) both trace gas ionized by young massive stars. Another method is to use the infrared (IR) flux as a tracer, since the UV radiation from young massive stars is also absorbed by surrounding dust and re-emitted in the IR. Combinations of various diffuse tracers (such as $24 \mu \mathrm{m}$ emission together with $\mathrm{H} \alpha$ ) are now commonly used. All these conventional methods usually agree but trace only the high-mass SFR, and require an extrapolation to lower mass stars.

For the Milky Way, we have the opportunity to directly estimate the SFR by counting individual young or forming stars, which if calibrated with caution is a preferred method as it accounts for the actual sites of star formation. With the Spitzer mid-infrared (MIR) survey "cores-to-disks" (Evans et al. 2009) the SFR of nearby star-forming regions was estimated to be $6.5 \times 10^{-6}-9.6 \times 10^{-5} M_{\odot} \mathrm{yr}^{-1}$ by counting young stellar objects (YSOs; down to low-mass objects), assuming an average mass of $0.5 M_{\odot}$ and a star formation duration of 2 Myr. For the first time, Robitaille \& Whitney (2010) calculated the total SFR of Milky Way by counting sources showing a MIR excess at IRAC wavelengths, using a population synthesis model to extrapolate the number of sources beyond the detection limit. They found an overall SFR for the Milky Way of $0.68-1.45 M_{\odot} \mathrm{yr}^{-1}$. This is in agreement with techniques based on diffuse emission tracers: indeed, Chomiuk \& Povich (2011) demonstrated that once the SFRs derived from the different methods are normalized to the same assumptions (for example, for the initial mass function (IMF)), the methods are all consistent with a value of $1.9 \pm 0.4 M_{\odot} \mathrm{yr}^{-1}$.

Yusef-Zadeh et al. (2009) calculated the SFR of the central molecular zone (CMZ) directly from their 599 classified YSOs with $24 \mu \mathrm{m}$ emission and found a rate of $0.14 M_{\odot} \mathrm{yr}^{-1}$. In order to derive this SFR, they determined stellar masses for each YSO, constructed a mass function, and fit a Kroupa IMF (Kroupa 2001) to the peak of the derived mass distribution in order to extrapolate the stellar mass below their sensitivity limit, then assumed the upper limit to the age of $1 \mathrm{Myr}$ for 213 of these objects. When comparing their derived SFR with values from indirect methods (e.g., free-free emission), large differences arise, up to a factor of 10, in contrast with the good agreement seen on the scale of the Milky Way as a whole. For example, Longmore et al. (2013) found, from their measurements of the total ionizing flux using the free-free emission, that the overall SFR of the CMZ is roughly $0.012-0.018 M_{\odot} \mathrm{yr}^{-1}$ for $|b| \leqslant 0.5$ and $0.06 M_{\odot} \mathrm{yr}^{-1}$ for $|b| \leqslant 1^{\circ}$, also covering areas outside the CMZ.

Whether the differences are real or due to issues with either the YSO-counting or the diffuse emission methods has not been established. The estimates based on YSOs could be heavily contaminated by reddened objects older than $1 \mathrm{Myr}$. In addition to these observational effects, the centimeter continuum and YSO counting methods may trace different timescales and thus would be expected to disagree if the star formation was episodic. In this paper, we aim to determine how much observational effects are able to contribute to this difference.

To distinguish embedded YSOs from more evolved objects, we applied the stage formalism introduced by Robitaille et al. (2006). With this formalism, true YSOs can be separated from more evolved objects. In this formalism, Stage 0/1 YSOs are very young and envelope-dominated, with $\dot{M}_{\text {gas }} / M_{\star}>$ $10^{-6} \mathrm{yr}^{-1}$. Assuming that the envelope infall rate goes down in time, this corresponds to an upper limit of the timescale of $1 \mathrm{Myr}$. Hence, since Yusef-Zadeh et al. (2009) assumed that their YSOs 

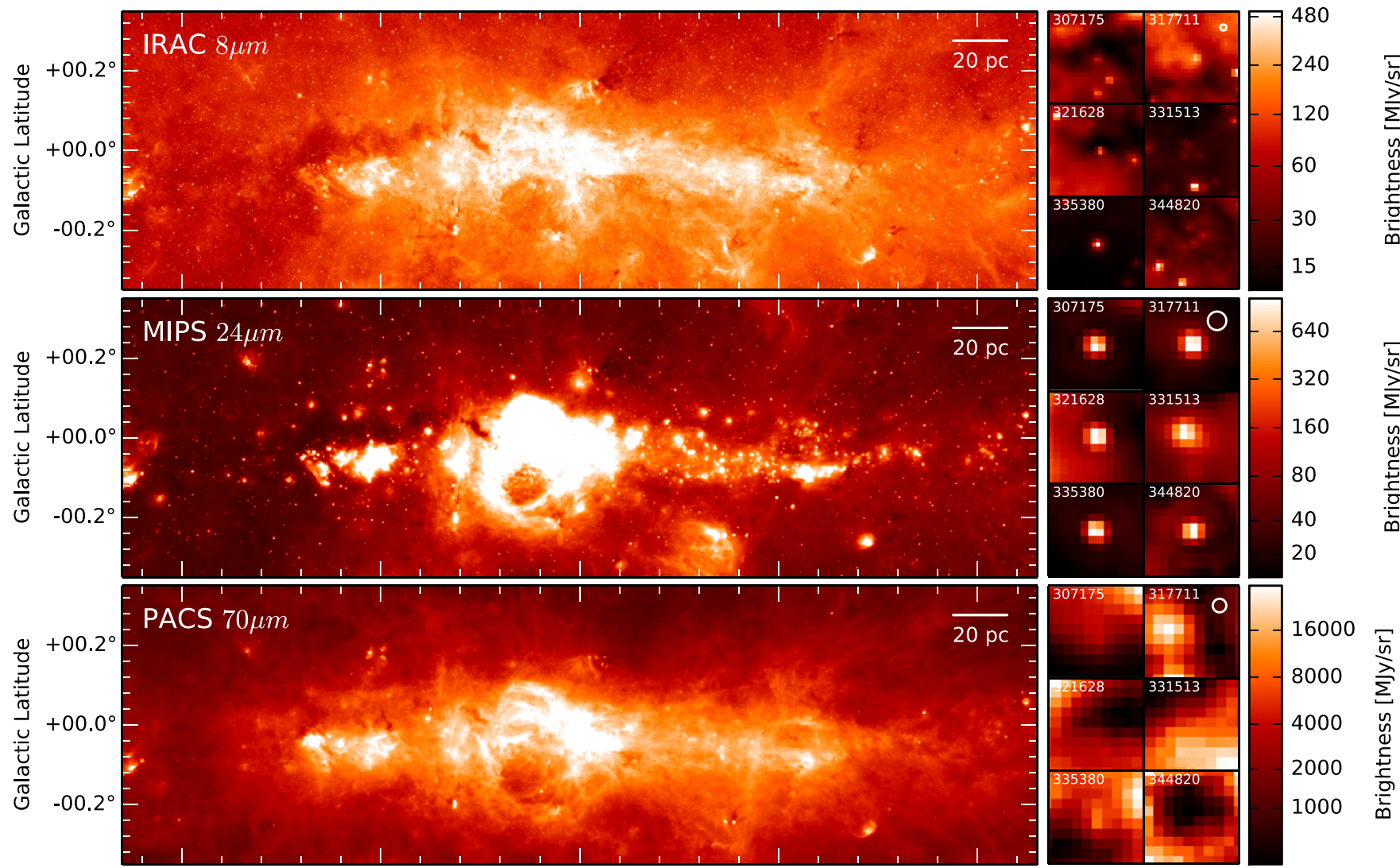

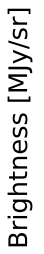
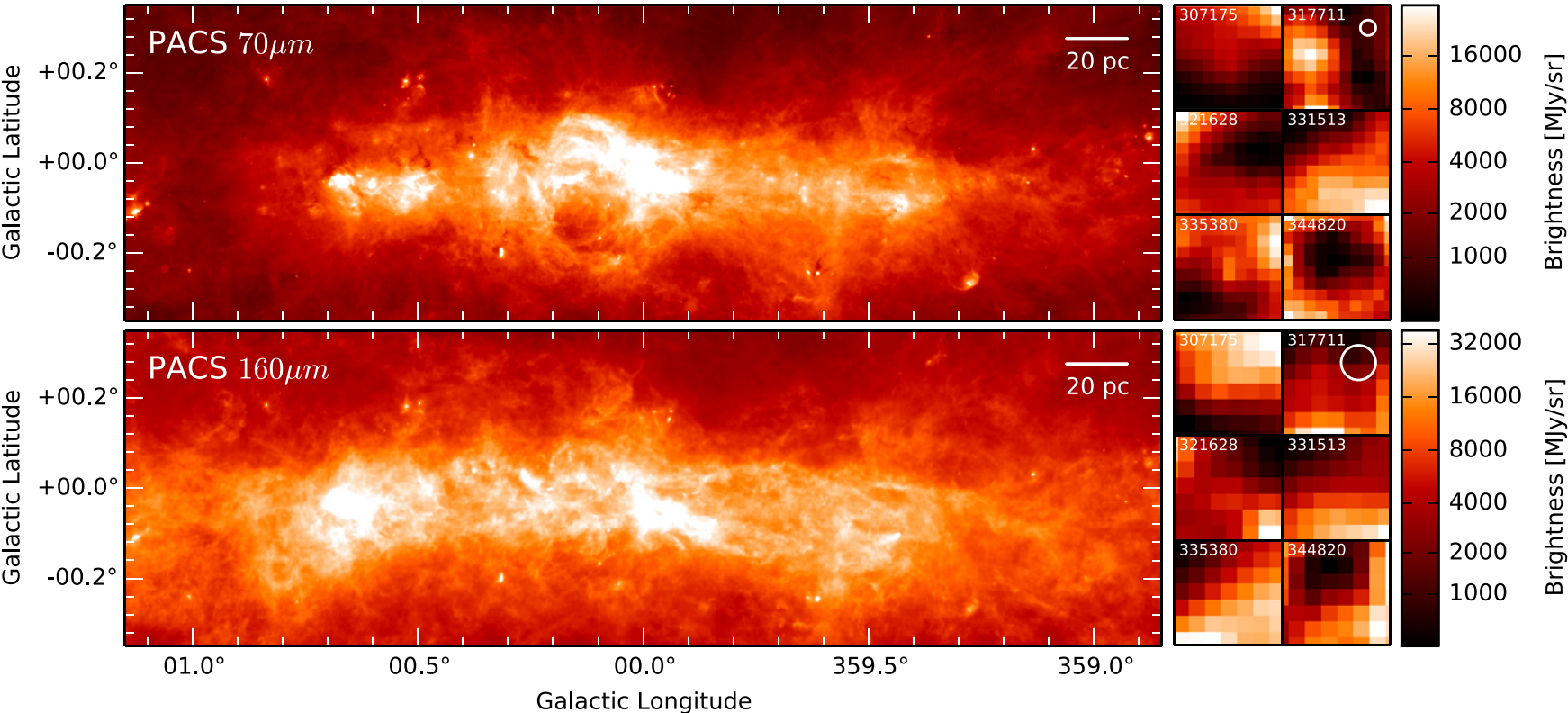

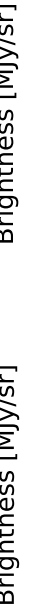

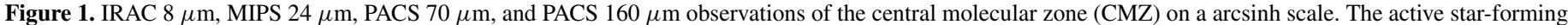

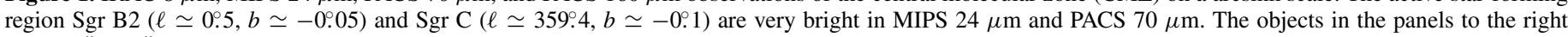

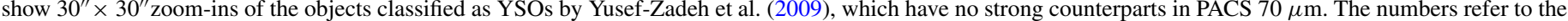

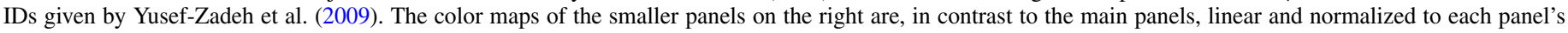
individual extrema. The PSF FWHMs are shown in the top right zoom panel for each wavelength.

were less than $1 \mathrm{Myr}$ old, these would be classified as Stage 0/1. In contrast, we group more evolved YSOs and main-sequence stars into the Stage $2+$ category, with $\dot{M}_{\text {gas }} / M_{\star}<10^{-6} \mathrm{yr}^{-1}$.

In Figure 1, we show the CMZ as observed by the Spitzer Space Telescope and the Herschel Space Observatory: the top panel shows the $8 \mu \mathrm{m}$ image observed with Spitzer's IRAC camera as a part of the GLIMPSE survey (Benjamin et al. 2003; Churchwell et al. 2009); below is the Spitzer MIPSGAL survey (Carey et al. 2009) using the $24 \mu \mathrm{m}$ band of the MIPS detector; and the two lower panels are FIR Herschel images (70 and $160 \mu \mathrm{m}$ ) from the Hi-GAL survey (Molinari et al. 2010) observed with the PACS detector. The Spitzer Space Telescope IR detectors IRAC (3.6, 4.5, 5.8, and $8.0 \mu \mathrm{m})$ and MIPS (24, 70 , and $160 \mu \mathrm{m}$ ) have a point-spread function (PSF) with full width 1".66, 1".72, 1".88, 1".98, and 6", 18", 40", respectively. By comparison, Herschel's PACS detector at 70, 100, and $160 \mu \mathrm{m}$ has a PSF with full width of about 4".4, 6.'1, and 9'.9. Hereafter, we will refer to the four bands shown in Figure 1 as IRAC $8 \mu \mathrm{m}$, MIPS $24 \mu \mathrm{m}$, PACS $70 \mu \mathrm{m}$, and PACS $160 \mu \mathrm{m}$, respectively.

By examining these observations, we found that some of the YSOs classified by Yusef-Zadeh et al. (2009) appear to have no or weak counterparts in PACS observations (see zoom-in panels in Figure 1), which is counterintuitive for YSOs. Most of these objects are located up to a Galactic longitude of about $\ell \simeq 359^{\circ} .5$, to the west of the Galactic center. In contrast, when looking at an active star-forming region (e.g., Sgr B2 at $\ell \simeq 0.5$ ), the star formation region as a whole is clearly seen in PACS.

Obscured main-sequence stars can mimic YSOs since the surrounding ambient dust is remitting the stellar flux in the IR (e.g., Whitney et al. 2013). Therefore, in this paper we set out to explore whether objects not seen at wavelength longer than $24 \mu \mathrm{m}$ may not be as young as $1 \mathrm{Myr}$ (as assumed by 
Yusef-Zadeh et al. 2009), and hence may not be members of the Stage 0/1 classification. However, we find instead that although non-detection at PACS wavelengths does not indicate whether or not a source is a YSO, its size at $24 \mu \mathrm{m}$ can be an age indicator.

To determine these results, we set up radiative transfer models and computed realistic synthetic observations of YSOs (Stage 0/1) and more evolved objects (Stage 2+) in different dust and density environments (Section 2). To determine, whether more evolved objects (Stage $2+$ ) could mimic YSOs (Stage 0/1), we compare the realistic synthetic observations directly with the observations and develop selection criteria that can help reduce contamination from evolved objects such as main-sequence stars (Section 3). In Section 4 we discuss the effects of masquerading main-sequence stars on the SFR of the CMZ. A summary and outlook is given in Section 5.

\section{MODELS}

To investigate whether main-sequence stars (Stage 2+) embedded in an ambient density medium could mimic deeply embedded YSOs (Stage 0/1) and match the measured brightness profile of the real observation in MIPS $24 \mu \mathrm{m}$, we performed radiative transfer calculations. We set up 660 models for different spectral types and evolutionary stages in an ambient medium with different dust and density properties. We used the threedimensional Monte Carlo radiative transfer code HyPERION (Robitaille 2011) to compute the temperature distribution and create synthetic images. By further modeling the effects of convolution with arbitrary PSFs, transmission curves, finite pixel resolution, noise and reddening, our radiative transfer models are then directly comparable to real observations. Our synthetic pipeline THE FLUXCOMPENSATOR will be made publicly available in the future. ${ }^{1}$

\subsection{Spectral Types and Stages of Evolution in an Ambient Density Environment}

We modeled main-sequence and young embedded $\mathrm{O}, \mathrm{B}$, and A stars, with temperatures ranging from 44,500 to $8200 \mathrm{~K}$, using in both cases the stellar atmosphere models of Castelli \& Kurucz (2004) as the central stars. We modeled the circumstellar density structure of the YSO models using a rotationally flattened envelope profile (Ulrich 1976), with gas infall rates from $3 \times 10^{-4} M_{\odot} \mathrm{yr}^{-1}$ to $3 \times 10^{-8} M_{\odot} \mathrm{yr}^{-1}$ determined from the scaling of the envelope density, an outer radius $1.5 \mathrm{pc}$, and a centrifugal radius at $100 \mathrm{AU}$. We assumed a gas-to-dust ratio of 100. The sublimation temperature, above which dust is removed, was set to $1600 \mathrm{~K}$. For all spectral types, we calculated 10 YSOs models and 1 additional model without an envelope (but with the constant density ambient medium), representing a main-sequence object in our simple approach. We use infall rate and stellar mass to classify every model as Stage $0 / 1$ or Stage 2+ using the Robitaille et al. (2006) formalism described in Section 1. The stellar data listed in Table 1 from Appendix E of Carroll \& Ostlie (1996) was used to determine the stellar radii and luminosities.

We placed all models within a surrounding ambient medium with a constant density $\rho_{0}$. We used three different ambient density environments $\rho_{0}=[1,3,10] \times 10^{-21} \mathrm{~g} \mathrm{~cm}^{-3}$, which are roughly within the number density range of $\left[10^{3} ; 10^{4}\right] \mathrm{cm}^{-3}$

\footnotetext{
1 For more information about HYPERION, and to sign up to be notified once the THE FLuxCOMPENSATOR package used here is available, visit http://www.hyperion-rt.org.
}

Table 1

Stellar Data Used in Radiative Transfer Setup

\begin{tabular}{lcccc}
\hline \hline SpT & $T$ & $L$ & $R$ & $M$ \\
& $(\mathrm{~K})$ & $\left(L_{\odot}\right)$ & $\left(R_{\odot}\right)$ & $\left(M_{\odot}\right)$ \\
\hline O5 & 44500 & 790000 & 15 & 60. \\
O6 & 41000 & 420000 & 13 & 37. \\
O8 & 35800 & 170000 & 11 & 23. \\
B0 & 30000 & 52000 & 8.4 & 17.5 \\
B1 & 25400 & 16000 & 6.5 & $13 .{ }^{\mathrm{a}}$ \\
B2 & 22000 & 5700 & 5.2 & $10 .^{\mathrm{a}}$ \\
B3 & 18700 & 1900 & 4.2 & 7.6 \\
B5 & 15400 & 830 & 4.1 & 5.9 \\
B8 & 11900 & 180 & 3.2 & 3.8 \\
A0 & 9520 & 54 & 2.7 & 2.9 \\
A5 & 8200 & 14 & 1.9 & 2.0 \\
\hline
\end{tabular}

Note. ${ }^{\text {a }}$ Values from interpolation of the stellar data.

found for the CMZ (see Molinari et al. 2011; Longmore et al. 2013). For the ambient medium, we also assumed a gas-to-dust ratio of 100 .

\subsection{Dust Properties}

For every combination of parameters, described in Section 2.1, we run the model for two different sets of dust properties. The first was the Milky Way dust from Draine (2003) with $R_{V}=5.5$ and $b_{c}=30 \mathrm{ppm}$, where $R_{V}$ is the ratio of the visual extinction to reddening magnitude, and $b_{c}$ is the concentration of carbon atoms in the medium. Weingartner \& Draine (2001) and Draine (2003) favor this combination for the Milky Way and point out that it best reproduces the conditions in the galactic center observed by Lutz et al. (1996). In the second configuration, in order to test the effect of polyaromatic hydrocarbons (PAHs), we additionally used the dust properties by Draine \& Li (2007) and as used in Robitaille et al. (2012) with a mixture of $5.9 \%$ ultra-small grains, $13.5 \%$ very small grains and $80.6 \%$ big grains with $R_{V}=3.1$ and $b_{c}=52 \mathrm{ppm}$.

\subsection{Realistic Synthetic Observations}

The synthetic images and spectral energy distributions (SEDs) computed by HYPERION are not directly comparable with photometric observations. In order to make these radiative transfer "observations" as realistic as possible, we developed a synthetic observations pipeline called THE FLUXCOMPENSATOR. In what follows we will describe it briefly.

For every model we produced realistic synthetic images as they were observed in four bands: the $K$ filter of the UKIDSS Galactic plane survey (Lucas et al. 2008), and the IRAC $8 \mu \mathrm{m}$, MIPS $24 \mu \mathrm{m}$, and PACS $70 \mu \mathrm{m}$ bands described in Section 1. Further information on these filters is provided in Table 2, and in the UKIDSS, Spitzer, and Herschel documentation. We convolved the synthetic images from HYPERION with the respective PSF after adjusting the pixel resolution. The original PSF files from Aniano et al. (2011) were used, except the MIPS $24 \mu \mathrm{m}$ PSF, which was directly derived from the observations.

We applied a filter convolution with the corresponding transmission functions from the detectors, and we accounted for reddening using the extinction law provided by Kim et al. (1994) and an optical extinction value of $A_{V}=20 \mathrm{mag}$. Estimates for the visual extinction toward the CMZ typically range from 20 to 30 mag (see Geballe et al. 2011), although the higher values likely include a contribution from extinction local to 
Table 2

Information About Telescopes and Detectors

\begin{tabular}{lcccc}
\hline \hline Name & $\begin{array}{c}\text { Zero-point } \\
(\mathrm{Jy})\end{array}$ & Filter & $\begin{array}{c}\text { Pixel Size } \\
(\operatorname{arcsec})\end{array}$ & PSF \\
\hline UKIDSS K & $631 .^{\mathrm{a}}$ & Hewett et al. (2006) & $0.4^{\mathrm{b}}$ & Gaussian $^{\mathrm{c}}$ \\
IRAC $8 \mu \mathrm{m}$ & $64.9^{\mathrm{c}}$ & Quijada et al. (2004) & $1.2^{\mathrm{c}}$ & Aniano et al. (2011) $^{\mathrm{d}}$ \\
MIPS $24 \mu \mathrm{m}$ & $7.17^{\mathrm{e}}$ & MIPS Handbook & $2.4^{\mathrm{e}}$ & Empirical $^{\mathrm{e}}$ \\
PACS $70 \mu \mathrm{m}$ & $0.78^{\mathrm{f}}$ & Herschel Science Center & $3.2^{\mathrm{h}}$ & ${\text { Aniano et al. }(2011)^{\mathrm{d}}}^{\mathrm{g}}$ \\
\hline
\end{tabular}

Notes.

${ }^{a}$ Hewett et al. (2006).

b UKIDSS Handbook: http://ukidss.org/technical/technical.html

${ }^{c}$ IRAC Handbook: http://irsa.ipac.caltech.edu/data/SPITZER/docs/irac

${ }^{\mathrm{d}}$ http://www.astro.princeton.edu/ ganiano/Kernels.html

e MIPS Handbook: http://irsa.ipac.caltech.edu/data/SPITZER/docs/mips/

${ }^{\mathrm{f}} \mathrm{http} / / / \mathrm{svo}$.cab.inta-csic.es/theory/fps/index.php?mode=browse \& gname $=$ Herschel

$\mathrm{g}$ https://nhscsci.ipac.caltech.edu/sc/index.php/Pacs/FilterCurves

h PACS Handbook: http://herschel.esac.esa.int/Docs/PACS/html/pacs_om.html

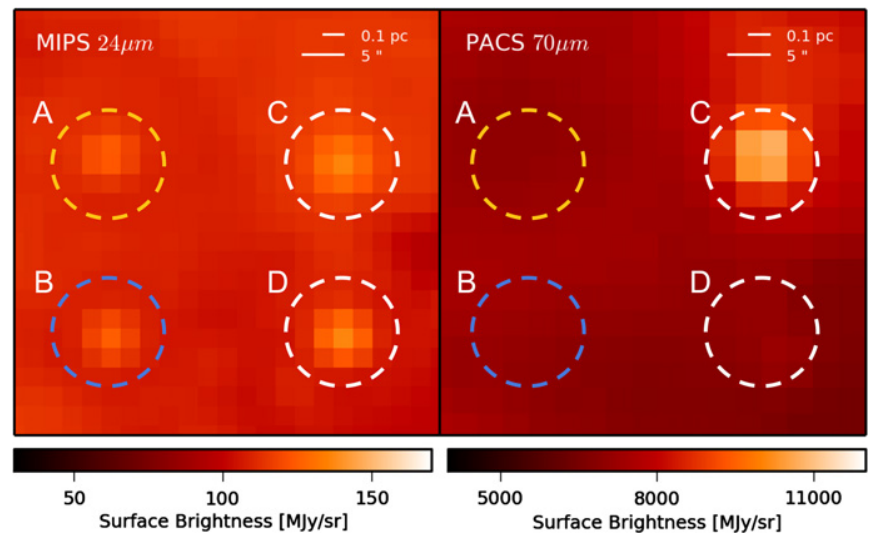

Figure 2. Patch of the CMZ observed by MIPS $24 \mu \mathrm{m}$ (left) and PACS $70 \mu \mathrm{m}$ (right). The real object (A) is marked with a yellow dashed circle, the synthetic Stage $2+$ source $(\mathrm{B})$ by a blue circle, and the two synthetic Stage 0/1 sources (C, D) by white circles. In MIPS $24 \mu \mathrm{m}$ the synthetic observations shown agree with the real observation. The synthetic Stage $2+$ source as well as the lower synthetic Stage 0/1 source agree with the PACS $70 \mu \mathrm{m}$ observation of the real source.

Sgr A; therefore, we assumed a value of $20 \mathrm{mag}$. Similar to Longmore et al. (2013) and Yusef-Zadeh et al. (2009), we assume a distance of $8.5 \mathrm{kpc}$ to the $\mathrm{CMZ}$. After generating realistic synthetic images for every model, we additionally measured the magnitudes and peak surface brightnesses. ${ }^{2}$ We calculated the total flux from the synthetic images with a field of view of $50^{\prime \prime} 4 \times 50^{\prime \prime} 4$ for UKIDSS $K$, IRAC $8 \mu \mathrm{m}$, and MIPS $24 \mu \mathrm{m}$. In all cases, the flux derived in this way is equivalent within $1 \%$ to the total integrated flux of the sources, that would be measured by standard photometry techniques such as PSF-fitting or aperture photometry (with aperture correction). We then converted these fluxes to magnitudes for the purpose of comparing these to observations. For the peak surface brightness we interpolate with two-dimensional cubic spline interpolation to extract the value at the real peak.

With the FLuxCOMPENSATOR, it is also possible to add noise. However, in order to account for a similar background as the one present in the real observations, we did not add synthetic noise. Instead it is possible to add the realistic synthetic image to the real observations (see Figure 2), so that the models

\footnotetext{
2 These and further parameters are provided in the Appendix (Table A1).
}

are directly comparable with real objects. This comparison is meaningful because for the ambient volume density we chose values comparable to measured average densities in the $\mathrm{CMZ}$ (see Section 2.1).

\section{RESULTS}

\subsection{Real Observations Versus Realistic Synthetic Observations}

In this section, we compare three model objects, as described in Section 2, added to observations to compare to a real source classified as a YSO. In Figure 2 we placed three synthetic observations (A0 Stage 2+, B5 and B8 Stage 0/1) next to a classified YSO of Stage $0 / 1$ by Yusef-Zadeh et al. (2009) at equatorial coordinates $\alpha_{\mathrm{J} 2000}=17^{\mathrm{h}} 44^{\mathrm{m}} 26.835, \delta_{\mathrm{J} 2000}=$ $-29^{\circ} 15^{\prime} 21^{\prime \prime}$.05 (yellow circle), which is clearly visible in MIPS $24 \mu \mathrm{m}$, but with no counterpart in the Herschel observations.

Our radiative transfer model of an embedded B5 YSO of Stage $0 / 1$ with $\dot{M}=3 \times 10^{-4} M_{\odot} \mathrm{yr}^{-1}$ (C: upper white circle) matches the real object in MIPS $24 \mu \mathrm{m}$ (A: yellow circle), but also produces a source detected in PACS $70 \mu \mathrm{m}$. On the other hand, the B8 Stage 0/1 model with $\dot{M}=10^{-4} M_{\odot} \mathrm{yr}^{-1}$ (D: lower white circle) has only a hardly detectable counterpart in PACS $70 \mu \mathrm{m}$, while matching the MIPS observation. Our model of a more evolved source, an A0 Stage $2+$ with $\dot{M}=$ $3 \times 10^{-7} M_{\odot} \mathrm{yr}^{-1}$, has no counterpart in PACS $70 \mu \mathrm{m}$ (B: blue circle). Therefore, the observed source can be explained by both Stage 0/1 and Stage 2+ models, and so may not be as young as 1 Myr.

\subsection{Evolution in the Spectral Energy Distribution}

In Figure 3, we show the evolution in the SED of a B0 star from deeply embedded to main-sequence object for three dust configurations: with regular dust and no extinction; with regular dust and extinction; and with PAH dust and extinction. Naturally, the extinction affects more strongly the near-infrared (NIR) bands. The PAHs add emission features in the MIR, but does not change too much above $24 \mu \mathrm{m}$. The FIR remains almost unaffected by both extinction and PAH dust emission.

Below we describe the evolution of the SED as a function of evolutionary stage. The evolution of the SED from a mainsequence source to a deeply embedded objects is best observed for regular dust and extinction (middle panel of Figure 3). For a main-sequence object (with no envelope, yellow line) surrounded by an ambient medium most of the mass is located at 


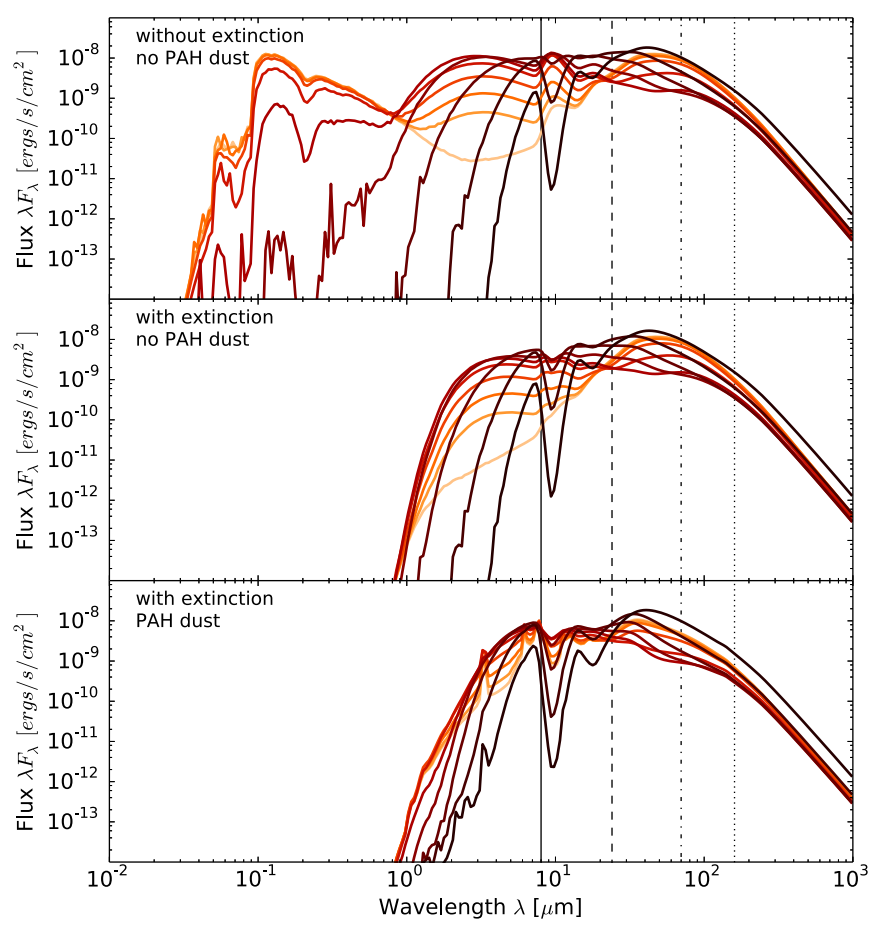

Figure 3. Evolution of the synthetic SEDs at different evolutionary stages of a B0 star embedded within an ambient medium of density $\rho_{0}=10^{-21} \mathrm{~g} \mathrm{~cm}^{-3}$ considering the effects of extinction and PAH dust. Vertical lines (solid: IRAC $8 \mu \mathrm{m}$, dashed: MIPS $24 \mu \mathrm{m}$, dot-dashed: PACS $70 \mu \mathrm{m}$, dotted: PACS $160 \mu \mathrm{m})$, SEDs from black to yellow $\left(3 \times 10^{-4} M_{\odot} \mathrm{yr}^{-1}, 10^{-4} M_{\odot} \mathrm{yr}^{-1}\right.$, $3 \times 10^{-5} M_{\odot} \mathrm{yr}^{-1}, 10^{-5} M_{\odot} \mathrm{yr}^{-1}, 3 \times 10^{-6} M_{\odot} \mathrm{yr}^{-1}, 10^{-6} M_{\odot} \mathrm{yr}^{-1}, 3 \times$ $10^{-7} M_{\odot} \mathrm{yr}^{-1}, 10^{-7} M_{\odot} \mathrm{yr}^{-1}, 3 \times 10^{-8} M_{\odot} \mathrm{yr}^{-1}$, main-sequence star with no envelope).

larger radii where the temperature is cold, and the mass of hotter material is low. This explains the lack of MIR emission. For the models with increasing infall rates, more material is added in the hotter regions closer to the star. As long as the dust is optically thin, the temperature of the dust in the inner regions stays constant, but the mass of the dust at these temperatures increases. The flux from this heated dust in the center is emitted in the NIR and MIR, which causes the rise at these wavelengths (e.g., infall rate $\left.10^{-6} M_{\odot} \mathrm{yr}^{-1}\right)$. NIR photons escape the system since the probability of re-absorption is too small. A higher accretion rate increases the envelope density, and the envelope starts to become optically thick to the stellar radiation at optical wavelengths. The temperature in the outer regions drops, resulting in a drop in the FIR emission. The emission in NIR and MIR goes down once the envelope is also no longer optically thin at that wavelength (see infall rate $>3 \times 10^{-6} M_{\odot} \mathrm{yr}^{-1}$ ), and the FIR emission rises again due to the absorbed radiation getting re-emitted.

For the six classified YSOs in the panels of Figure 1, we now plot the measured SEDs in Figure 4. We used the available IRAC fluxes published in the point-source catalog of Ramírez et al. (2008), and extracted the MIPS $24 \mu \mathrm{m}$ from the pointsource catalog provided by Hinz et al. (2009). These measured fluxes were the same as used by Yusef-Zadeh et al. (2009). We computed the $5 \sigma$ upper limits for PACS $70 \mu \mathrm{m}$ (triangles) after removing the background. We assumed an average error of $10 \%$ in the fluxes as used by Robitaille et al. (2007). The black solid line shows the fit $\chi_{\text {best }}^{2}$ with the lowest $\chi^{2}$. The gray solid lines represent the synthetic model SEDs for which $\left(\chi^{2}-\chi_{\text {best }}^{2}\right) / N_{\text {sample }}<3$ as defined in Robitaille et al. (2007). Object 307175 is very well fitted by a B5 Stage 0/1 model. For object 331513, we found from inspecting the images that

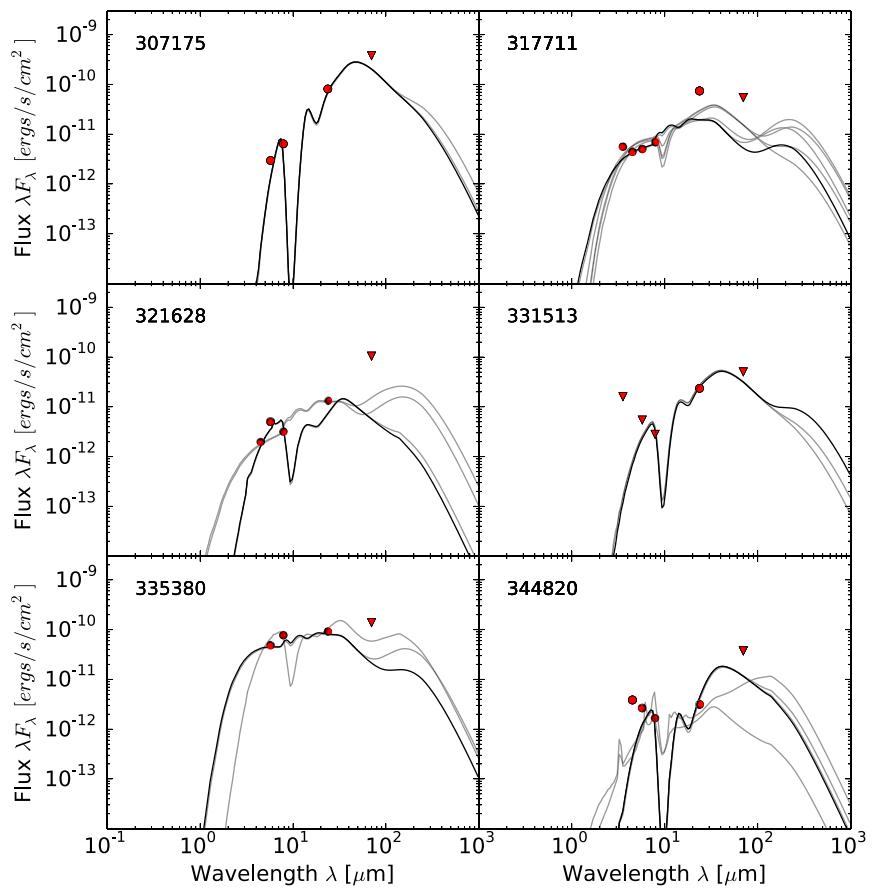

Figure 4. Real SEDs (circles) of six classified YSOs shown in Figure 1. The black and gray solid lines represent the synthetic SEDs from the modeling with the best and acceptable $\chi^{2}$ fits, respectively. The triangles represent $5 \sigma$ upper limits in PACS $70 \mu \mathrm{m}$ (and for object 331513 also in the IRAC bands).

the IRAC $8 \mu \mathrm{m}$ source may not be related to the MIPS $24 \mu \mathrm{m}$ source since it is not well aligned, so we use the IRAC fluxes as $5 \sigma$ upper limits (triangles). Object 344820 is not well fit. It has a very weak MIPS $24 \mu \mathrm{m}$ source. We remeasured its total flux and found 21.5 instead of $26.7 \mathrm{mJy}$, which improves the fit marginally, but since the IRAC fluxes are still not well fit, and are typical of an unextincted source, they may originate from an unrelated foreground object. Objects 317711, 321628, 335380, and 344820 can be either fitted by Stage $0 / 1$ or Stage $2+$ objects of spectral type B5, B8, A0, A5 and B8, B5, A0, respectively. Hence, SED fitting alone cannot be used to distinguish between true YSOs and more evolved objects.

\subsection{Evolution in the Radial Profiles}

By inspecting the YSOs classified by Yusef-Zadeh et al. (2009) in the $24 \mu \mathrm{m}$ images, we found that some objects appear to be resolved, i.e., some sources are slightly larger than point sources. Therefore, we explore the radial brightness profiles of our synthetic images in MIPS $24 \mu \mathrm{m}$.

In Figure 5, a compilation of normalized profiles of the realistic synthetic observations are shown. The dashed line represents the PSF of a perfect point source. Its FWHM is roughly 6". Without an envelope (Figure 5(a)) the sources become more extended for higher temperature stars. The low density of the surrounding medium enhances this effect (Figure 5(b)), while the presence of circumstellar dust reduces the width of the profile (Figure 5(c)). Resolved sources could therefore be earlier type stars and/or stars embedded in a low-density environment (either circumstellar and/or ambient medium).

\subsection{Distinguishing Embedded YSOs from More Evolved Objects}

In this section, based on the observational properties of our models such as MIPS $24 \mu \mathrm{m}$ magnitude, MIPS $24 \mu \mathrm{m}$ angular size and MIR color, we explore how to distinguish between true 

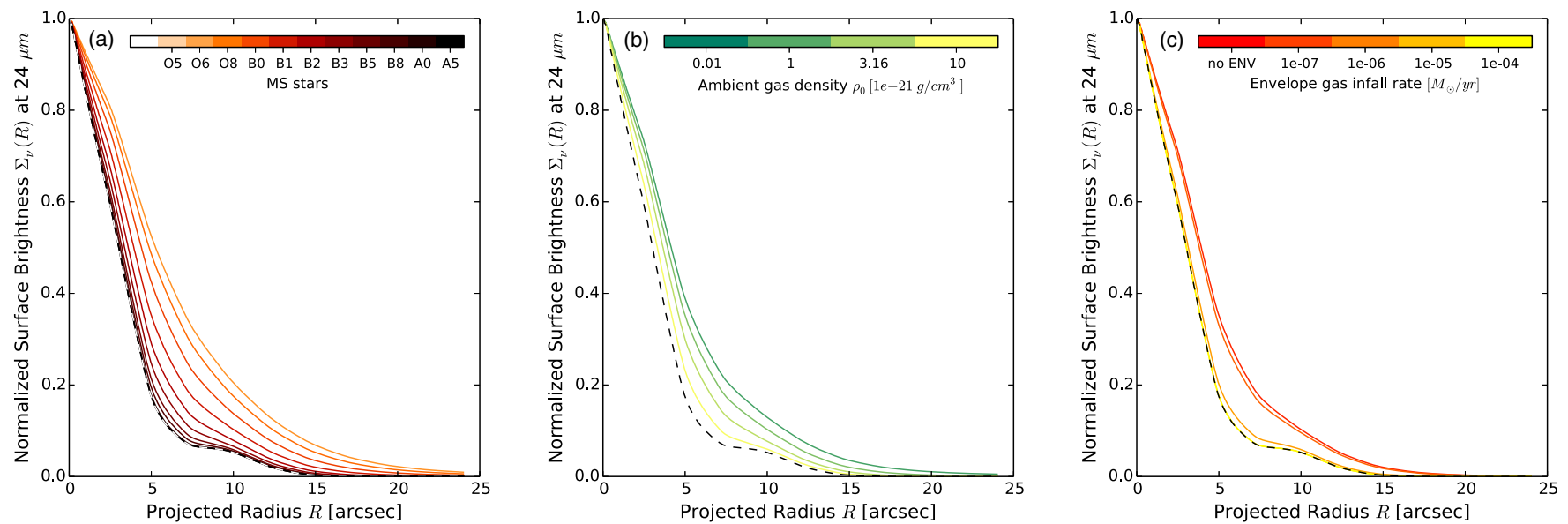

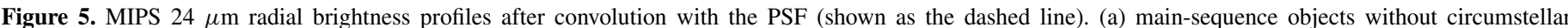

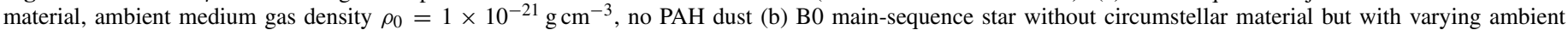

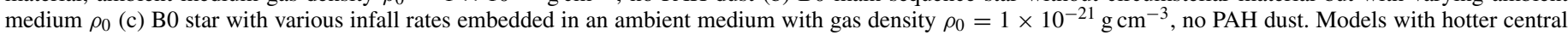

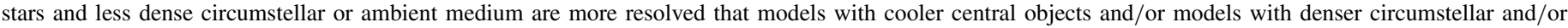
ambient medium.

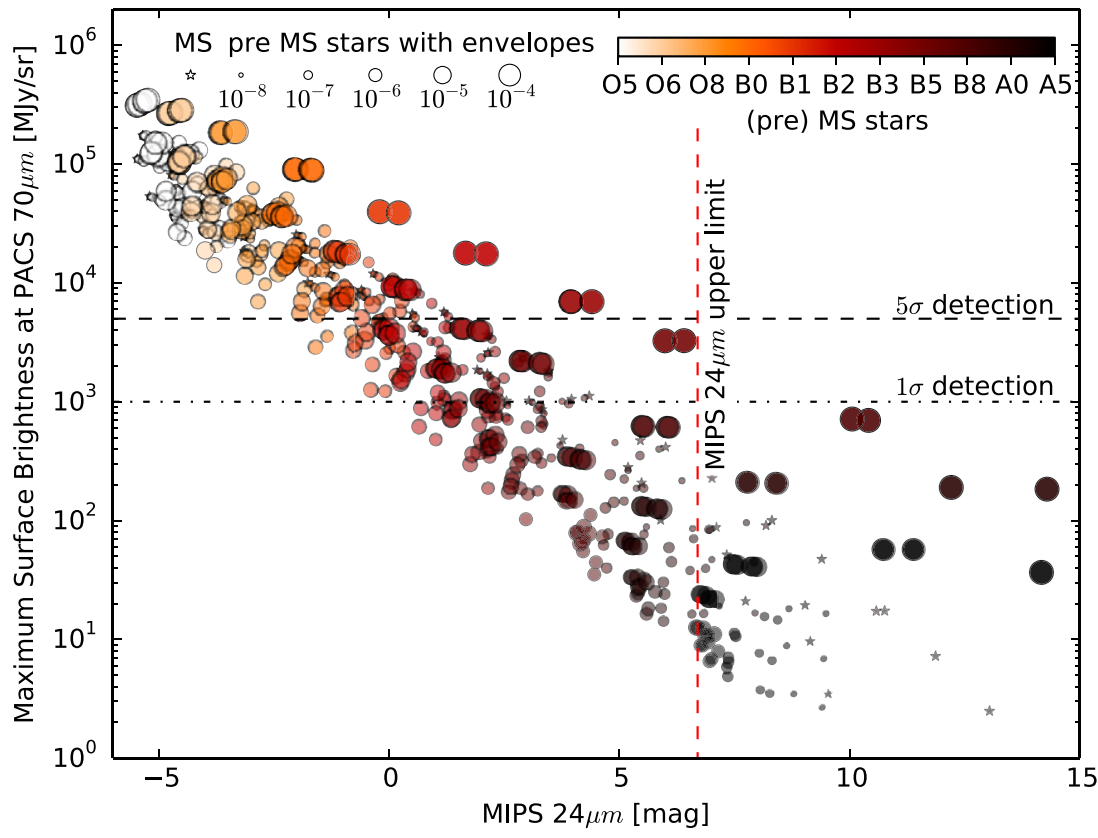

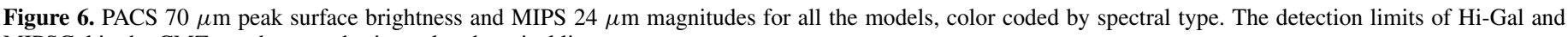
MIPSGal in the CMZ are shown as horizontal and vertical lines.

YSOs (Stage 0/1) from more evolved objects (Stage 2+) in the CMZ.

\subsubsection{Detection in MIPS $24 \mu \mathrm{m}$ and PACS $70 \mu \mathrm{m}$}

Using the source counts as a function of magnitude of the point-source catalogs, we determined approximate detection limits for the western part of the CMZ, where most of the YSOs classified by Yusef-Zadeh et al. (2009) are located. For UKIDSS $K$, IRAC $8 \mu \mathrm{m}$, and MIPS $24 \mu \mathrm{m}$, we found, respectively, upper limits of $14.8 \mathrm{mag}, 8.9 \mathrm{mag}$, and $6.0 \mathrm{mag}$ for a completeness of $90 \%$, and $15.5 \mathrm{mag}, 9.8 \mathrm{mag}$, and $6.7 \mathrm{mag}$ for a completeness of $50 \%$, within an error of about $0.25 \mathrm{mag}$, which is the bin width used to construct the magnitude histograms. For PACS $70 \mu \mathrm{m}$, we estimated a $5 \sigma$ surface brightness detection to be at roughly $5000 \mathrm{MJy} \mathrm{sr}^{-1}$. In Figure 6, we show the PACS $70 \mu \mathrm{m}$ peak surface brightness versus the MIPS $24 \mu \mathrm{m}$ magnitude for the 660 modeled objects, indicating the different spectral types and envelope infall rates. A total of 567 model objects can be detected in MIPS $24 \mu \mathrm{m}$ as indicated by the vertical dashed line in Figure 6. The MIPS $24 \mu \mathrm{m}$ detection limit corresponds to a completeness limit of B5 in spectral type. On the other hand, the PACS $70 \mu \mathrm{m} 5 \sigma$ detection limit (also shown in Figure 6) translates into a completeness limit of $\mathrm{O} 8$ in spectral type, but also some evolutionary stages of B0-B3 objects can be $5 \sigma-$ detected in PACS $70 \mu \mathrm{m}$, while later types show no $5 \sigma$-detected counterparts.

In Figure 7(a) the models from Figure 6 are classified with the stage formalism described in Section 1. As we can see, Stage $0 / 1$ models lie above and below the $5 \sigma$-detection limit in PACS $70 \mu \mathrm{m}$. Therefore, detection or non-detection of sources at PACS $70 \mu \mathrm{m}$ does not allow us distinguish true YSOs from more evolved objects in the CMZ, disproving the hypothesis put 

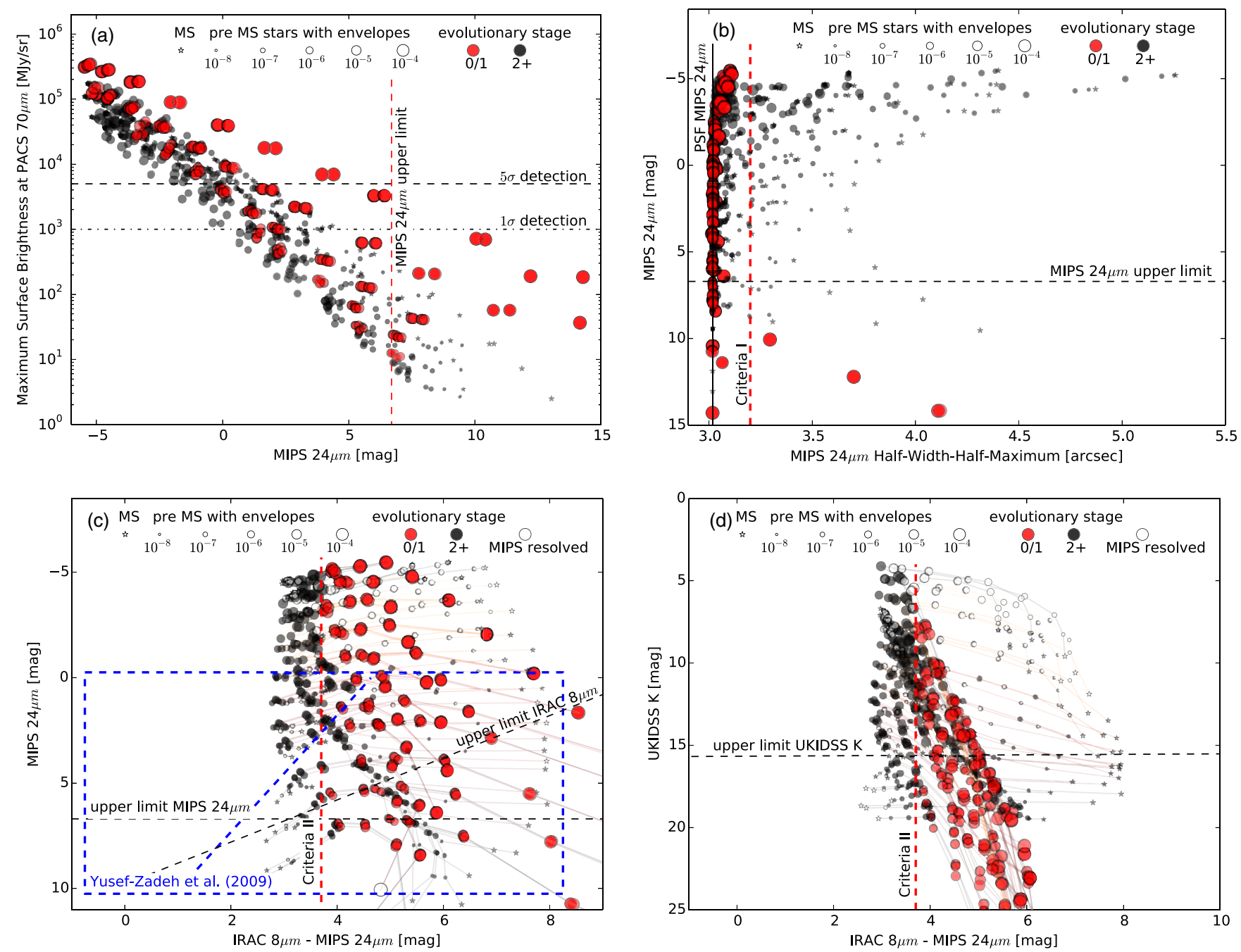

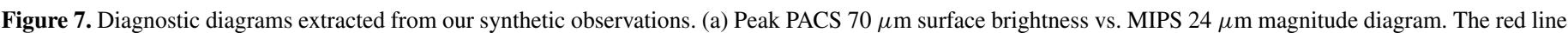

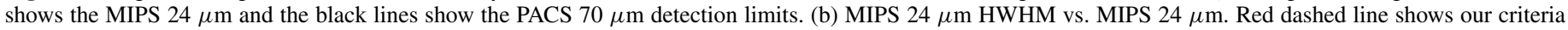

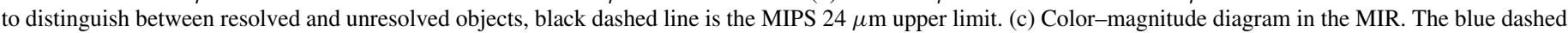

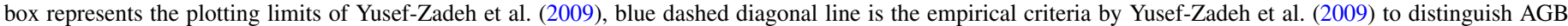

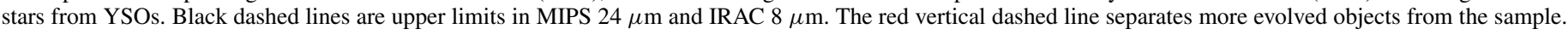

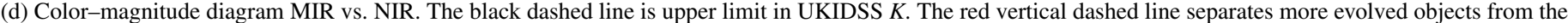
sample (red: Stage 0/1, black: Stage 2+, white: MIPS resolved).

forward in Section 1 that objects only seen at MIPS $24 \mu \mathrm{m}$ and not detected at PACS $70 \mu \mathrm{m}$ would not be YSOs.

\subsubsection{Half Width at Half Maximum in MIPS $24 \mu \mathrm{m}$}

To explore the effects in the radial profiles described in Section 3.3, we need the half-width at half-maximum (HWHM) of all models in MIPS $24 \mu \mathrm{m}$. We developed a tool which can fit PSF models for extended sources, which consist of Gaussian profiles convolved with the MIPS $24 \mu \mathrm{m}$ PSF. The fit can be carried out manually in order to ensure an optimal fit in regions of complex background. The profile with the best fit is used to calculate the "observed" HWHM, which is roughly $\sqrt{\mathrm{HWHM}_{\mathrm{PSF}}^{2}+\mathrm{HWHM}_{\mathrm{Gauss}}^{2}}$. We fit the synthetic sources with 100 profiles with combined HWHMs ranging from $3^{\prime \prime}$ to $6^{\prime \prime}$ (1-2 HWHM). With this PSF-fitting method it was also possible to extract total integrated fluxes. In Section 3.5 we use this technique on real observations.

In Figure 7(b), we plot the MIPS $24 \mu \mathrm{m}$ magnitude versus the MIPS $24 \mu \mathrm{m}$ HWHM. About half of the objects lie exactly on the PSF (HWHM = 3.'02) and are therefore unresolved. We find that most resolved objects have an inverse timescale $\dot{M}_{\text {gas }} / M_{\star}$ less than $10^{-6} \mathrm{yr}^{-1}$ and are therefore not likely to be as young as assumed by, e.g., Yusef-Zadeh et al. (2009).

We adopt the stage formalism to explore whether the objects in the vicinity of the PSF can be disentangled. We found that 228 of the 660 model objects are in fact still in the envelope-dominated phase (Stage 0/1). We define a HWHM threshold of 3 ". 2 to separate resolved objects (HWHM $\geqslant 3$ ".2) from unresolved objects (HWHM < 3.'2). There are only nine resolved Stage 0/1 model objects, and these are all below the MIPS $24 \mu \mathrm{m}$ detection limit. Therefore, most of the resolved objects (165 of 174) are more evolved Stage 2+. A total of 486 objects are unresolved and include objects from both stages of evolution (219 Stage 0/1, 267 Stage 2+). Note that while seeing an extended source at $24 \mu \mathrm{m}$ likely indicates that a source is not truly young, unresolved sources can still be ambiguous.

\subsubsection{MIR and NIR Color-Magnitude Diagrams}

In what follows, we now have a closer look at the 481 unresolved model objects using color-magnitude diagrams in 
order to understand to what extent we can distinguish embedded YSOs from more evolved objects. Figure 7(c) presents the IRAC $8 \mu \mathrm{m}-$ MIPS $24 \mu \mathrm{m}$ versus MIPS $24 \mu \mathrm{m}$ color-magnitude diagram. Yusef-Zadeh et al. (2009) used an empirical criterion (see blue dashed diagonal line in Figure 7(c)) to distinguish asymptotic giant branch (AGB) field stars from YSOs. Our modeling shows that this criterion alone (without accounting for resolved and unresolved objects) is not effective to separate true YSOs from more evolved objects, since these overlap in color-magnitude space. The evolutionary tracks in the color-magnitude diagram can be explained similarly to the SED evolution described in Section 3.2. Although there is some overlap, sources with IRAC $8 \mu \mathrm{m}-$ MIPS $24 \mu \mathrm{m}<3.7$ mag are always more evolved.

One can see that the MIR color-magnitude diagram alone (without accounting for resolved and unresolved objects) is not capable of completely distinguishing between more evolved and deeply embedded YSOs. Combining bands in the NIR and the MIR seems more promising if the detection in $K$ is sensitive enough, as can be noted in Figure 7(d), which shows the IRAC $8 \mu \mathrm{m}-$ MIPS $24 \mu \mathrm{m}$ versus UKIDSS $K$ color-magnitude diagram. The $K$ band is dominated by stellar emission that is simply extincted, so it directly probes the envelope column density. Since the column density is very different between the main-sequence stars and ambient medium compared to with envelopes, all envelope dominated objects fall in one distinctive region in the diagram.

\subsubsection{Criteria to Select True YSOs}

In summary, we have shown that the following criteria can be used to preferentially select truly young Stage 0/1 YSOs, based on the MIPS $24 \mu \mathrm{m}$ size and IRAC $8 \mu \mathrm{m}-$ MIPS $24 \mu \mathrm{m}$ color:

$$
\begin{aligned}
& \text { I : } \operatorname{MIPS} 24 \mu \mathrm{m} \text { HWHM }<3^{\prime \prime} .2 \\
& \text { II : } \text { IRAC } 8 \mu \mathrm{m}-\text { MIPS } 24 \mu \mathrm{m}>3.7 \mathrm{mag} \text {. }
\end{aligned}
$$

Of the 660 model objects, 343 match these criteria. Of these, 219 are true YSOs models and 124 are models of more evolved objects. Nine objects are misclassified as more evolved but are true YSOs and lie below the MIPS $24 \mu \mathrm{m}$ detection limit. The remaining 308 are all models of more evolved objects. In summary, using these criteria on our models results in

$$
\begin{aligned}
& 33.2 \% \text { : correctly classified Stage } 0 / 1 \text { objects, } \\
& 46.7 \% \text { : correctly classified Stage } 2+\text { objects, } \\
& 18.8 \% \text { : misclassified as Stage } 0 / 1, \\
& 1.4 \% \text { : misclassified as Stage } 2+\text {. }
\end{aligned}
$$

When including protoplanetary disks $\left(r_{\max }=1000 \mathrm{AU}\right)$ to our YSO circumstellar geometry setup the above criteria are mostly unchanged. Overall, models with disks are slightly less extended due to the increased mass of circumstellar material in the center. Therefore, the selection criterion I in MIPS $24 \mu \mathrm{m}$ is not quite as successful as for the runs without disks. For models with a disk, the classification fractions are as follows:

$33.2 \%$ : correctly classified Stage $0 / 1$ objects,

$33.3 \%$ : correctly classified Stage $2+$ objects,

$32.1 \%$ : misclassified as Stage 0/1,

$1.4 \%$ : misclassified as Stage $2+$.

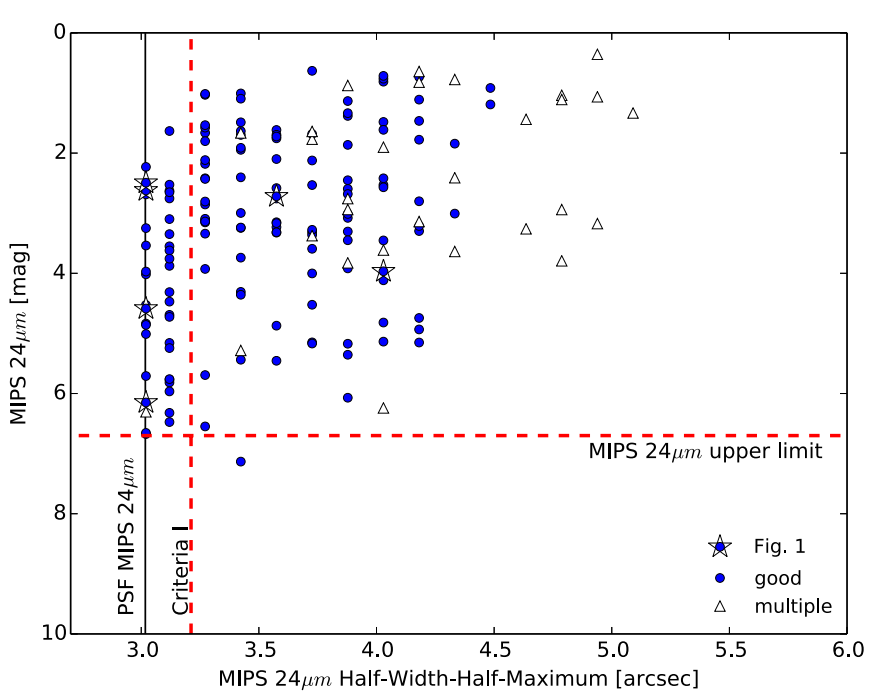

Figure 8. Observational counterpart to Figure 7(b). Measured sizes of sample points of good quality (blue circles) and others which appear to be multiple sources (white triangles) from the Yusef-Zadeh et al. (2009) sample. The sources of bad quality are not included. Points with white stars surrounded by blue circles represent the measurements of the six objects showed in Figure 1.

\subsection{Correcting Previously Estimated SFR}

With these criteria it is possible to revise SFR calculations from the literature. Here we investigate the 213 sources classified as Stage 0/1 by Yusef-Zadeh et al. (2009). We use the PSF-fitting tool described in Section 3.4.2 with 20 extended models with total HWHMs ranging from $3^{\prime \prime}$ to $6^{\prime \prime}$. We used fewer profiles than for the synthetic observations, because the fitting is done by hand for real observations. The measuring tool is robust to distinguish between unresolved and resolved objects. The error increases with increasing size in real observations, but sizes smaller than HWHM $<3$ ". 5 appear to be robust. We also estimate the total flux of the objects. The values of the magnitudes suffer from errors depending on the background. In Figure 8 we plot our measured sizes and magnitudes.

By visually inspecting the MIPS $24 \mu \mathrm{m}$ images, we noted first that about $31.0 \%$ of the sources are likely unreliable, of which $56 \%$ correspond to parts of diffuse emission erroneously fitted as point sources in the original catalogue and $44 \%$ of the sources show evidence of multiplicity.

Further, and more importantly, 32.4\% of the sample have HWHM $>3$ ".5 and would clearly be resolved according to our criteria. When we use our original threshold of 3 .'2, $49.3 \%$ of the objects have a larger HWHM, but this may include objects that appear to be larger due to noise, so that this value should be treated more cautiously. This means that at least $63.4 \%(32.4 \%+$ $31.0 \%)$ and maybe $80.3 \%(49.3 \%+31.0 \%)$ or more of the Yusef-Zadeh et al. (2009) sources may therefore not be YSOs, lowering the SFR by a factor of three or more and bringing it closer to the value of the SFR estimated from free-free emission (see Longmore et al. 2013).

Given the issues with spurious sources mentioned above, a careful characterization of each source at MIPS $24 \mu \mathrm{m}$ and IRAC $8 \mu \mathrm{m}$ is therefore needed in future to pin down the SFR in the $\mathrm{CMZ}$ more accurately.

\section{DISCUSSION}

Our "realistic synthetic observations" from radiative transfer modeling (see Section 2) have shown that more evolved objects (i.e., main-sequence stars) in a low-density ambient density 
medium could mimic YSOs for all spectral types discussed in this paper. We found that Stage 2+ objects with spectral types later than B3, detected in MIPS $24 \mu \mathrm{m}$, are predicted to have no $5 \sigma$-observable counterpart in PACS $70 \mu \mathrm{m}$, similar to some of the objects classified as YSOs by Yusef-Zadeh et al. (2009).

Unresolved MIPS $24 \mu \mathrm{m}$ sources could be heavily embedded objects (Stage 0/1) or more evolved Stage 2+, while resolved objects are most likely Stage $2+$ objects. This means that resolved model objects detected in MIPS $24 \mu \mathrm{m}$ in the CMZ are more evolved and therefore likely older than 1 Myr. All these arguments then indicate some objects classified in the CMZ are not very young embedded YSOs (i.e., less than $1 \mathrm{Myr}$ ) as previously thought. Therefore, the SFR of $0.14 M_{\odot} \mathrm{yr}^{-1}$ (YusefZadeh et al. 2009) is likely overestimated.

\subsection{Stellar Distribution from the IMF}

To get an idea of how many main-sequence stars of various spectral types should be in the CMZ, we performed simple estimations using a Kroupa (2001) IMF. Assuming a constant SFR of $0.01 M_{\odot} \mathrm{yr}^{-1}$ (see Longmore et al. 2013), roughly four O stars and 5183 B stars (including and earlier than B5) should be located in the CMZ. We note that in this IMF calculation the ages of the stars are equally distributed. Since the lifetime of a MIPS $24 \mu \mathrm{m}$ detectable B star lies, as mass decreases (B0-B5, $\left.M \in[17.5,5.9] M_{\odot}\right)$, between $8 \mathrm{Myr}$ and $113 \mathrm{Myr}$, only a few objects should be primordial (Stage 0/1, smaller than 1 Myr), while the majority would be in the main-sequence phase (Stage $2+$ ). Not necessarily all 5138 B stars (including and earlier than B5) could be observed in MIPS $24 \mu \mathrm{m}$, since sources in lower ambient densities environments cannot produce strong MIR emission in order to be detected.

\subsection{Origin of the Main-sequence Stars}

In the CMZ there are many objects with MIPS $24 \mu \mathrm{m}$ emission west from the Galactic center, which appear not to be part of an active star-forming region analogous to Sgr B2 or Sgr C. Our results show that some of those may be in fact more evolved objects (i.e., main-sequence stars) and not true YSOs.

However, more evolved objects with ages of several Myr will not have formed at the observed spot. One could envisage a scenario in which an $\mathrm{OB}$ association formed at the current location of Sgr B2, then got disrupted at a later time while orbiting the Galactic center. For example, an object observed at 41.6 or 103 pc from the Galactic center with an average orbital speed of about $140 \mathrm{~km} \mathrm{~s}^{-1}$ (Sofue 2013) has an orbital timescale of 4.5 Myr, thus all more evolved objects with spectral types earlier than B5 will have completed at least one orbit around the Galactic center since their birth.

\subsection{Giants in the $C M Z$}

It is also possible that giants could mimic YSOs. An et al. (2011) found that there are supergiants in the CMZ. Evolved objects like supergiants should not produce $\mathrm{H}$ II regions. There is not a large overlap with the Yusef-Zadeh et al. (2009) sample of YSOs but still when recalculating the SFR one should consider these objects, because contamination of the sample would overestimate the SFR. We produced synthetic observation of supergiants $^{3}$ from spectral type $\mathrm{B} 0$ to $\mathrm{M} 0$ and found that they are distributed amongst resolved sources and could be therefore also be removed by our selection criteria. Further, in the NIR/MIR color space (e.g., Figure 7(d)) most supergiants are redder than

\footnotetext{
The setup and photometric data is provided in the Appendix.
}

Stage 0/1 sources but brighter than 12 mag in $K$ band and 8 mag in IRAC $8 \mu \mathrm{m}$, and therefore would be easily distinguishable in color space.

\subsection{Predictions for High-resolution Millimeter Observations}

We now briefly describe whether high-resolution millimeter observations could help us to distinguish between the mainsequence stars (Stage 2+) and true YSOs (Stage 0/1). In particular, one would expect that the YSOs will show much more dense dust/gas in the central regions. Millimeter observations of reclassified Stage 0/1 and Stage 2+ sources could help us to constrain our predictions, and reduce the number of Stage 2+ objects misclassified as YSOs. In Figure 9, our $0.9 \mathrm{~mm}$ prediction of ALMA observations using HYPERION and CASA of a B5 star ${ }^{3}$ at all evolutionary stages is presented (dust continuum with a bandwidth of $7.5 \mathrm{GHz}$, total observing time $1200 \mathrm{~s}$, beam size $\sim 0$.'5). The four models to the right with the highest infall rate are Stage 0/1 sources. Stage 0/1 objects are much brighter and have much more flux on the small scales, while for the most evolved ones, there is very little dense material so most of the emission is extended or not detected. Our analysis of the synthetic ALMA images show that it would be possible to use millimeter observations to distinguish between the mainsequence stars and the YSOs.

\section{SUMMARY}

With our realistic synthetic observation from radiative transfer modeling, we have shown that some of the classified YSOs (Yusef-Zadeh et al. 2009) in the CMZ may not necessarily be as young as previously thought (i.e., less than $1 \mathrm{Myr}$ ). In addition, we have shown that some of the observed objects can be better explained by more evolved objects such as main-sequence stars in a constant density interstellar medium. We found that

1. detection/non-detection in PACS $70 \mu \mathrm{m}$ is not a reliable handle to distinguish true YSOs from more evolved objects;

2. resolved, extended objects in MIPS $24 \mu \mathrm{m}$ are unlikely to be deeply embedded YSOs and are therefore not truly young.

These findings lead us to believe that the SFR in the CMZ estimated by directly counting YSOs (Yusef-Zadeh et al. 2009) is overestimated by at least a factor of three (and potentially up to a factor of five). A lower SFR for the CMZ would be in better agreement with estimates from free-free emission (e.g., Longmore et al. 2013). By producing synthetic observations of our YSO models, we have shown that high-resolution dust continuum observations with ALMA could in future help to provide a more definite classification of the YSO candidates.

We thank the referee for a constructive report that helped us improve the clarity and the strength of the results presented in our paper. This work was carried out in the Max Planck Research Group Star formation throughout the Milky Way Galaxy at the Max Planck Institute for Astronomy. C.K. is a fellow of the International Max Planck Research School for Astronomy and Cosmic Physics (IMPRS) at the University of Heidelberg, Germany, and acknowledges support. This research made use of Astropy, a community-developed core Python Astronomy (Astropy Collaboration 2013) and matplotlib (Hunter 2007), a python plotting library. 


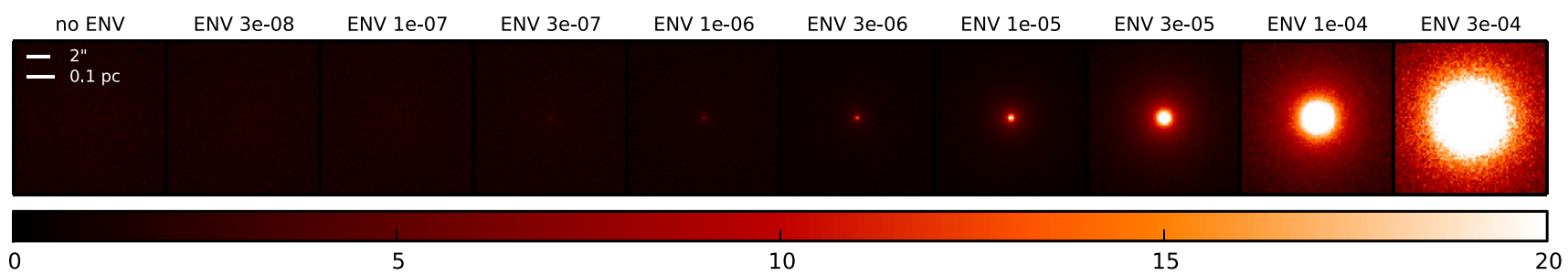

Synthetic HYPERION image at $0.92 \mathrm{~mm}[\mathrm{MJy} / \mathrm{sr}]$

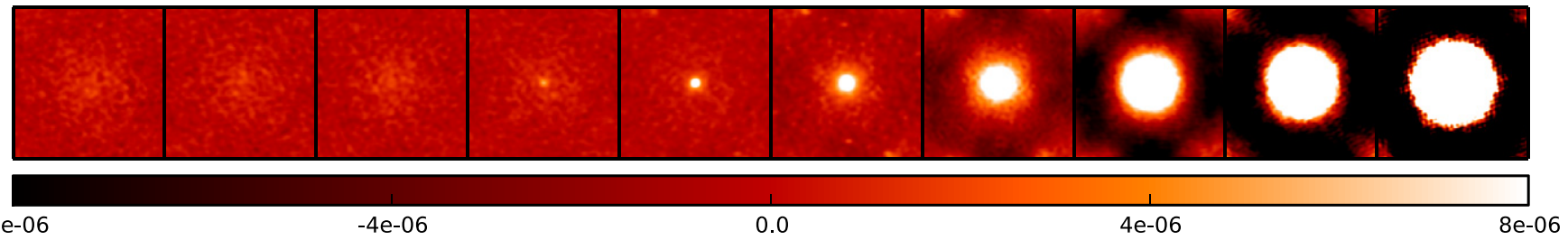

Synthetic ALMA image at $0.92 \mathrm{~mm}$ [Jy/beam]

Figure 9. Millimeter observation observed through a perfect interferometer of a B5 star (top) and synthetic ALMA observations (bottom). True YSO (Stage 0/1) model objects are much brighter and have much more flux on the small scales, while for the most evolved ones, there is very little dense material so most of the emission is extended or not detected. Six models from the left: Stage 2+, four models from the right: Stage 0/1.

Table A1

Parameters and Measurements from our Models

\begin{tabular}{|c|c|c|c|c|c|c|c|c|c|}
\hline Star & $\begin{array}{l}\text { Infall Rate } \\
\left(M_{\odot} \mathrm{yr}^{-1}\right)\end{array}$ & Stage & $\begin{array}{l}\text { Ambient Density } \\
\left(\mathrm{g} \mathrm{cm}^{-3}\right)\end{array}$ & PAH Dust & $\begin{array}{c}K 2.2 \mu \mathrm{m} \\
(\mathrm{mag})\end{array}$ & $\begin{array}{l}\text { IRAC } 8 \mu \mathrm{m} \\
\quad(\mathrm{mag})\end{array}$ & $\begin{array}{l}\text { MIPS } 24 \mu \mathrm{m} \\
\quad(\mathrm{mag})\end{array}$ & $\begin{array}{l}\text { PACS } 70 \mu \mathrm{m} \\
\left(\mathrm{MJy} \mathrm{sr}^{-1} \text { ) }\right.\end{array}$ & $\begin{array}{l}\text { HWHM } 24 \mu \mathrm{m} \\
\quad(\operatorname{arcsec})\end{array}$ \\
\hline$\ldots$ & $\ldots$ & $\ldots$ & $\ldots$ & $\ldots$ & $\ldots$ & $\ldots$ & $\ldots$ & $\ldots$ & $\ldots$ \\
\hline B5 & 0 & $2+$ & $1 \times 10^{-21}$ & $\ldots$ & 16.29 & 13.21 & 5.46 & 471.53 & 3.11 \\
\hline B5 & $3 \times 10^{-8}$ & $2+$ & $1 \times 10^{-21}$ & $\ldots$ & 15.68 & 9.57 & 4.91 & 452.39 & 3.07 \\
\hline B5 & $1 \times 10^{-7}$ & $2+$ & $1 \times 10^{-21}$ & $\ldots$ & 14.98 & 8.43 & 4.14 & 407.34 & 3.03 \\
\hline B5 & $3 \times 10^{-7}$ & $2+$ & $1 \times 10^{-21}$ & $\ldots$ & 14.04 & 7.46 & 3.31 & 305.25 & 3.02 \\
\hline B5 & $1 \times 10^{-6}$ & $2+$ & $1 \times 10^{-21}$ & $\ldots$ & 13.08 & 6.82 & 2.85 & 188.92 & 3.02 \\
\hline B5 & $3 \times 10^{-6}$ & $2+$ & $1 \times 10^{-21}$ & $\ldots$ & 12.54 & 6.66 & 2.66 & 220.14 & 3.02 \\
\hline B5 & $1 \times 10^{-5}$ & $0 / 1$ & $1 \times 10^{-21}$ & $\ldots$ & 13.47 & 6.88 & 2.16 & 492.63 & 3.02 \\
\hline B5 & $3 \times 10^{-5}$ & $0 / 1$ & $1 \times 10^{-21}$ & $\ldots$ & 18.81 & 7.51 & 1.97 & 1070.34 & 3.02 \\
\hline B5 & $1 \times 10^{-4}$ & $0 / 1$ & $1 \times 10^{-21}$ & - & 38.45 & 9.73 & 2.84 & 2205.17 & 3.02 \\
\hline B5 & $3 \times 10^{-4}$ & $0 / 1$ & $1 \times 10^{-21}$ & $\ldots$ & 88.48 & 16.45 & 5.99 & 3257.50 & 3.02 \\
\hline$\ldots$ & $\ldots$ & $\ldots$ & $\ldots$ & $\ldots$ & $\ldots$ & $\ldots$ & $\ldots$ & $\ldots$ & $\ldots$ \\
\hline
\end{tabular}

(This table is available in its entirety in a machine-readable form.)

\section{APPENDIX}

Here we present input (spectral type, envelope infall rate, stage, ambient medium, dust type) and output (magnitude in UKIDSS $2.2 \mu \mathrm{m}$, IRAC $8 \mu \mathrm{m}$, and MIPS $24 \mu \mathrm{m}$ and the maximum surface brightness in PACS $70 \mu \mathrm{m}$, MIPS $24 \mu \mathrm{m}$ HWHM) parameters of the 660 models of YSOs and mainsequence stars in an ambient density environment as well as the models of the supergiants.

\section{REFERENCES}

An, D., Ramírez, S. V., Sellgren, K., et al. 2011, ApJ, 736, 133

Aniano, G., Draine, B. T., Gordon, K. D., \& Sandstrom, K. 2011, PASP, 123,1218

Astropy Collaboration, Robitaille, T. P., Tollerud, E. J., et al. 2011, A\&A, 558, A33

Benjamin, R. A., Churchwell, E., Babler, B. L., et al. 2003, PASP, 115, 953

Calzetti, D. 2013, in Secular Evolution of Galaxies, ed. J. Falcón-Barroso \& J. H. Knapen (Cambridge: Cambridge Univ. Press), 419

Carey, S. J., Noriega-Crespo, A., Mizuno, D. R., et al. 2009, PASP, 121, 76

Carroll, B. W., \& Ostlie, D. A. 1996, An Introduction to Modern Astrophysics (Reading, MA: Addison Wesley)

Castelli, F., \& Kurucz, R. L. 2004, arXiv:astro-ph/0405087

Chomiuk, L., \& Povich, M. S. 2011, AJ, 142, 197
Churchwell, E., Babler, B. L., Meade, M. R., et al. 2009, PASP, 121, 213

Draine, B. T. 2003, ARA\&A, 41, 241

Draine, B. T., \& Li, A. 2007, ApJ, 657, 810

Evans, N. J., II, Dunham, M. M., Jørgensen, J. K., et al. 2009, ApJS, 181,321

Geballe, T. R., Najarro, F., Figer, D. F., Schlegelmilch, B. W., \& de La Fuente, D. 2011, Natur, 479, 200

Hewett, P. C., Warren, S. J., Leggett, S. K., \& Hodgkin, S. T. 2006, MNRAS, 367,454

Hinz, J. L., Rieke, G. H., Yusef-Zadeh, F., et al. 2009, ApJS, 181, 227

Hunter, J. D., et al. 2007, Computing in Science \& Engineering, 9, 90

Kim, S.-H., Martin, P. G., \& Hendry, P. D. 1994, ApJ, 422, 164

Kroupa, P. 2001, MNRAS, 322, 231

Longmore, S. N., Bally, J., Testi, L., et al. 2013, MNRAS, 429, 987

Lucas, P. W., Hoare, M. G., Longmore, A., et al. 2008, MNRAS, 391, 136

Lutz, D., Feuchtgruber, H., Genzel, R., et al. 1996, A\&A, 315, L269

Molinari, S., Bally, J., Noriega-Crespo, A., et al. 2011, ApJL, 735, L33

Molinari, S., Swinyard, B., Bally, J., et al. 2010, PASP, 122, 314

Quijada, M. A., Marx, C. T., Arendt, R. G., \& Moseley, S. H. 2004, Proc. SPIE, 5487,244

Ramírez, S. V., Arendt, R. G., Sellgren, K., et al. 2008, ApJS, 175, 147

Robitaille, T. P. 2011, A\&A, 536, A79

Robitaille, T. P., Churchwell, E., Benjamin, R. A., et al. 2012, A\&A, 545, A39

Robitaille, T. P., \& Whitney, B. A. 2010, ApJL, 710, L11 
Robitaille, T. P., Whitney, B. A., Indebetouw, R., \& Wood, K. 2007, ApJS, 169,328

Robitaille, T. P., Whitney, B. A., Indebetouw, R., Wood, K., \& Denzmore, P. 2006, ApJS, 167, 256

Sofue, Y. 2013, PASJ, 65, 118
Ulrich, R. K. 1976, ApJ, 210, 377

Weingartner, J. C., \& Draine, B. T. 2001, ApJ, 548, 296

Whitney, B. A., Robitaille, T. P., Bjorkman, J. E., et al. 2013, ApJS, 207, 30

Yusef-Zadeh, F., Hewitt, J. W., Arendt, R. G., et al. 2009, ApJ, 702,178 